\title{
GENERATION OF FOCUSED SURFACE WAVES WITH A SOLID WEDGE
}

\author{
Abdullah ATALAR and Hayrettin KÖYMEN $\dagger$ \\ Electrical and Electronics Engineering Department, Bilkent University \\ $\dagger$ Electrical and Electronics Engineering Department, Middle East Technical University \\ Ankara, Turkey.
}

\begin{abstract}
Focusing of surface acoustic waves on material surfaces was achieved by axicons in liquid immersion. The system consisted of a plane acoustic wave generator obliquely insonifying a cylindrical mirror placed perpendicularly on the surface to be examined. A similar axicon can be implemented with a solid cylindrical structure which does not require an immersion liquid. The lower cap of a solid cylinder is placed on the surface of the solid object, while the upper cap, where a shear wave transducer is placed, is inclined with respect to the axis of the cylinder. Shear waves generated by the transducer are reflected from the free cylindrical surface of the solid. The reflected waves are incident on the bottom surface at a critical angle and couple to the object surface as surface waves to converge to a diffraction limited focus. The cylindrical solid material must be chosen from low shear wave velocity materials. The critical angle determines the inclination of the top surface and it is found from the solution of reflection problem for shear waves at a solidsolid interface with a slippery boundary condition. The focusing system is implemented successfully with lucite as the solid and aluminum as the object material
\end{abstract}

\section{INTRODUCTION}

Use of focused surface acoustic waves (SAW) for imaging purposes have been previously demonstrated $[1,2]$. The system consists of a parabolic cylindrical mirror surface insonified obliquely by a plane sound wave in a liquid medium. The reflected waves form a conical wavefront. If the conical wavefronts propagating in the liquid medium are incident at a planar solid interface at the Rayleigh critical angle, all of the power in the conical wavefront will be transferred to a leaky SAW. The surface waves will focus to a diffraction limited spot before they completely leak back into the liquid medium. Any inhomogeneity at the focal point will cause a reflection of the surface waves, and the reflected SAW will find its way back to the transducer which originally created them. Used in pulse echo mode, it is possible to build an imaging system with this configuration [3]. Such an imaging system requires a liquid immersion medium.

Using the same principle, it is also possible to build a SAW focusing setup without an immersion liquid. Such a system will be essentially a solid wedge transducer $[4,5]$ with a built-in focusing mechanism as explained below.

\section{FOCUSING SYSTEM}

Fig. 1 illustrates the setup used. It consists of a solid cylindrical block

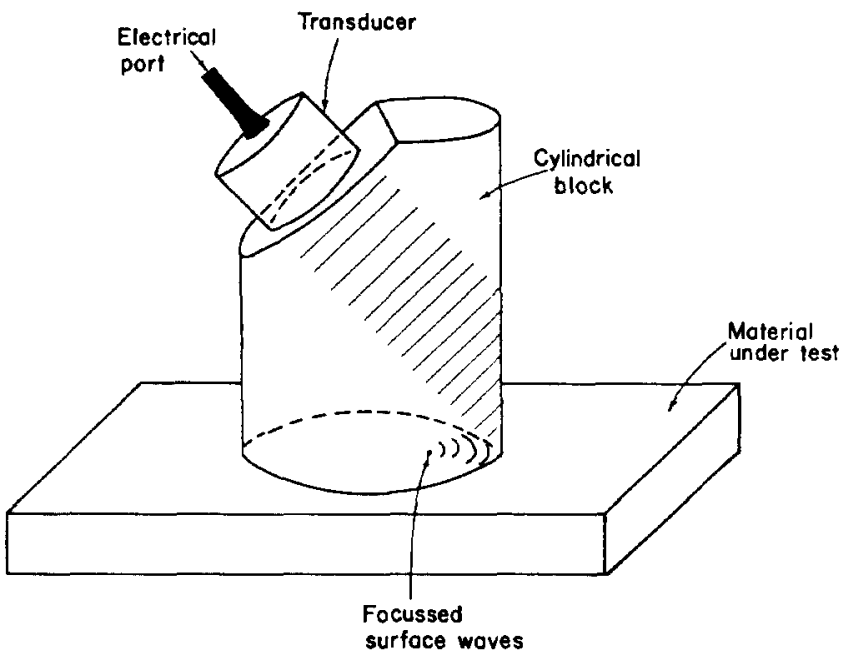

Figure 1: Arrangement used to focus SAW at the object surface

with an orthogonal planar surface on one end and with an angled planar surface on the other end. A shear wave transducer is placed on the angled surface so that the shear waves generated by the transducer insonify the cylindrical surface formed by the solid-air interface of the block. When a small portion of the circular cylindrical surface is utilized, it approximates well a parabolic cylindrical surface. No mode conversion at reflection exists, because, typically, for this surface the incidence angle is above the critical angle for longitudinal wave excitation [6]. Fig. 2 shows the plots of the reflection coefficient at a stress-free solid boundary for some solids. The critical angle is about 31.3 degrees for lucite and 20.5 degrees for lead. The reflected shear waves form a conical wavefront in the solid block as before [2]. The axis of the generated conical wavefront will lie at a distance approximately half the cylinder radius from the reflecting surface. If the solid block is placed on a solid object surface to be examined, the conical wavefronts will impinge on the solid-solid interface formed by the solid block and the object. Typically, one would use a liquid couplant gel between the solid block and the object, in which case, the interface formed is a solid-solid interface with a slippery boundary. As we will show later, it is possible to excite SAW at the interface provided that the shear wave velocity in the solid block is less - preferably, by a factor of two or more - than the wave velocity on the interface. Moreover, the inclination of the up- 


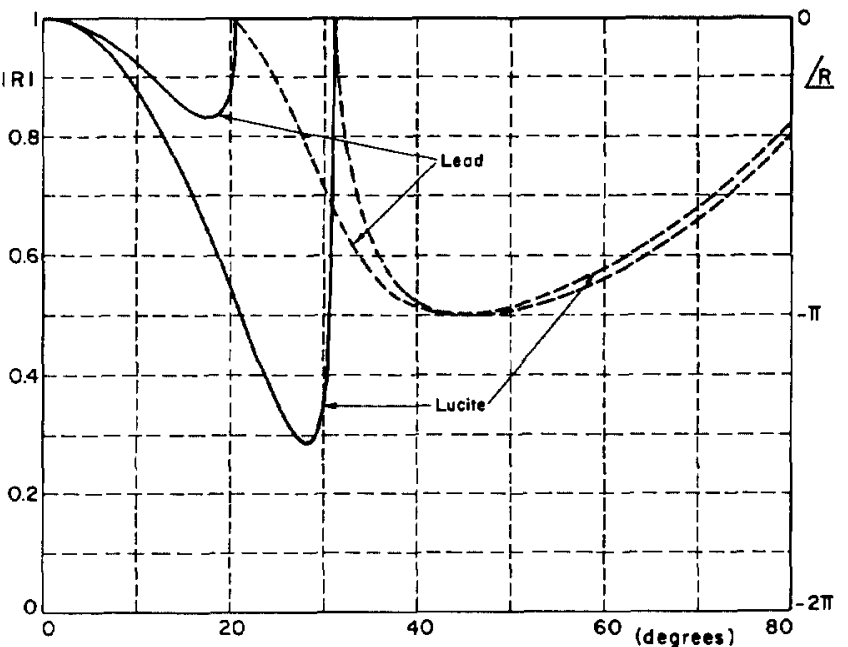

Figure 2: Reflection coefficient magnitude (solid curve) and phase (dashed curve) as a function of incidence angle for a stress-free lucite and lead boundaries

per surface of the block must be selected so that, the shear waves are incident at the interface at a particular angle in such a way to excite this wave. The polarization of shear waves must be vertical, because a horizontally polarized shear wave will not couple to the object material through a slippery boundary. It is appropriate to choose a low shear velocity material for the block to keep the inclination of the upper surface at a practical value. Use of longitudinal waves. instead of shear waves, would have required a solid material whose longitudinal velocity is less than the wave velocity at the interface. This is a more stringent requirement to satisfy and such material combinations which give realizable incidence angles are hard to come by.

Excited SAW will converge to a diffraction limited spot, because the intersection of a conical surface with the planar object surface is always circular. If there is a reflector at the focal point, the waves will return through the same path to the shear wave transducer which originally generated them. When it is used in pulse-echo mode, it is possible to detect the return echo. Note that, not all of the incident energy will be converging to the focal point, some of it will leak back into the solid block in the form of shear waves before it reaches the focus. Similarly, not all of the reflected SAW will be collected by the transducer, because some of the waves will keep propagating in the form of SAW

We have solved the shear-wave incidence acoustic reflection problem at a solid-solid interface with a slippery boundary (See Fig. 3). Isotropic and nonpiezoelectric solids are assumed. The boundary conditions are the continuity of normal particle velocity and the normal traction force. The components of the traction force parallel to the surface on both sides must be zero because of the slippery condition. From these four boundary conditions, it is possible to find an analytic solution for this problem and the reflection coefficient for shear waves can be written as

$$
\mathcal{R}=\frac{N}{D}-1
$$

with

$$
N=4 \sqrt{\left(k_{S}^{2}-k_{x}^{2}\right)\left(k_{L}^{\prime 2}-k_{x}^{2}\left(k_{L}^{2}-k_{x}^{2}\right)\right.}
$$

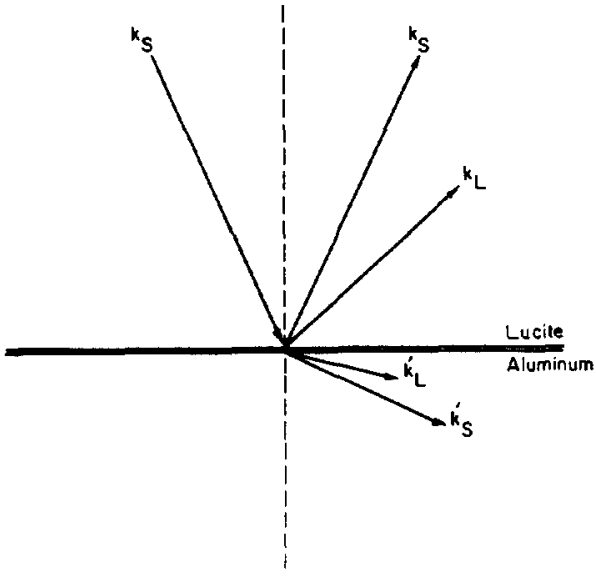

Figure 3: Geometry of the reflection problem at a solid-solid (lucite-aluminum) interface with a slippery boundary

$$
\begin{aligned}
& \text { and } \\
& \qquad \begin{aligned}
D= & 2 \sqrt{\left(k_{L}^{\prime 2}-k_{x}^{2}\right)\left(k_{L}^{2}-k_{x}^{2}\right)}\left(\sqrt{k_{S}^{2}-k_{x}^{2}}+\frac{c_{44}^{\prime} k_{S}^{2}}{c_{44} k_{S}^{\prime 2}} \sqrt{k_{S}^{\prime 2}-k_{x}^{2}}\right) \\
& +\left(\frac{k_{S}^{2}}{2 k_{x}^{2}}-1\right)\left(\frac{c_{11}}{c_{44}} k_{L}^{2}-2 k_{x}^{2}\right) \sqrt{k_{L}^{\prime 2}-k_{x}^{2}} \\
& +\frac{k_{S}^{2}}{k_{S}^{\prime 2}}\left(\frac{k_{S}^{2}}{2 k_{x}^{2}}-1\right)\left(\frac{c_{11}^{\prime}}{c_{44}} k_{L}^{2}-2 \frac{c_{44}^{\prime}}{c_{44}} k_{x}^{2}\right) \sqrt{k_{L}^{2}-k_{x}^{2}}
\end{aligned}
\end{aligned}
$$

where $\mathcal{R}$ is the reflection coefficient of vertically polarized shear waves, subscripts $S$ and $L$ refer to shear and longitudinal waves, primed and unprimed variables refer to the object and block medium, respectively. $k$ 's represent wavenumbers, $k_{x}$ is the component of the wave vector along the interface and $c$ 's are elastic constants.

Lucite has a low shear velocity, $1287 \mathrm{~m} / \mathrm{sec}$ [7], therefore it is appropriate for most object materials. We have plotted the magnitude and phase of the reflection coefficient as a function of incidence angle in Fig. 4 for lucite-aluminum interface. From the magnitude curve, three critical angles are apparent: For angles smaller than $\theta_{c L}^{\prime}=\sin ^{-1}\left(k_{L}^{\prime} / k_{S}\right)$ three refracted waves - a reflected longitudinal wave, transmitted longitudinal and shear waves - will exist in addition to the reflected shear wave. For angles exceeding $\theta_{c L}^{\prime}$ no longitudinal waves in aluminum can exist. Above $\theta_{c S}^{\prime}=\sin ^{-1}\left(k_{S}^{\prime} / k_{S}\right)$ the shear waves in the aluminum can no longer be excited, and finally beyond $\theta_{c L}=\sin ^{-1}\left(k_{L} / k_{S}\right)$ a longitudinal wave reflection in the lucite can not occur. The phase transition at $\theta_{C R}=31^{\circ}$ indicates that a wave at interface is excited. This angle corresponds to a wave velocity of about $2500 \mathrm{~m} / \mathrm{sec}$ at the interface, considerably less than the SAW velocity on the free aluminum surface. It is seen that this wave is excited at an angle slightly higher than $\theta_{c L}$. The velocity of this wave is primarily determined by the shear wave to longitudinal velocity ratio in the lucite, not by the SAW velocity in the aluminum. This is a rather interesting wave excited when a vertically polarized shear wave is incident at a slippery solid-solid interface. At this incidence angle, all the waves except the shear wave in the lucite are evanescent. Hence, the generated wave is a leaky wave much like the SAW excited on a liquid-solid interface.

The Schoch Displacement, $\Delta_{S}$, can be related to the rate of change of reflection coefficient phase with incidence angle [8]. This value can be readily determined from the analytical expression [9]:

$$
\Delta_{S}=-\left.\frac{\partial}{\partial k_{x}} L \mathcal{R}\right|_{k_{x}=k_{S} \sin \theta_{c R}}=-\left.\frac{1}{k_{S} \cos \theta_{c R}} \frac{\partial}{\partial \theta} L \mathcal{R}\right|_{\theta=\theta_{c R}}
$$



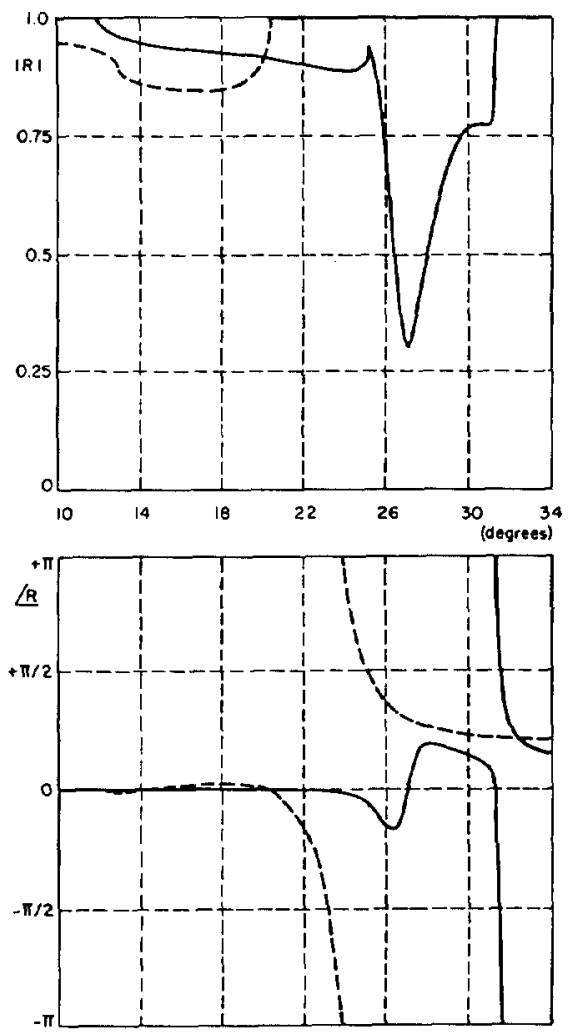

Figure 4: Magnitude (a) and phase (b) of the reflection coefficient as a function of incidence angle at the lucite-aluminum (solid curve) and lead-aluminum (dashed curve) interfaces for the slippery boundary case

where $\angle \mathcal{R}$ indicates the phase of the reflection coefficient and $\theta_{\mathcal{C} R}$ is the critical angle. $\Delta_{S}$ is a factor which shows the rate of decay of SAW and it is important for optimization of focusing system efficiency. We have used Eq 1 and 2 to calculate a number of cases. Table 1 summarizes the results. For optimum efficiency, the radius of the cylinder block

\begin{tabular}{|l|c|c|}
\hline Material Pair & $\theta_{C R}$ (degrees) & $\Delta_{S} / \lambda_{S}$ \\
\hline Lucite-Al & 31.48 & 280 \\
Lucite-Fe & 31.30 & 8380 \\
Lucite-Ni & 31.31 & 4190 \\
Pb-Al & 23.95 & 26 \\
Pb-Fe & 21.32 & 181 \\
\hline
\end{tabular}

Table 1: Critical angle and Schoch Displacement for a number of material combinations

must be related to the Schoch Displacement by [2]

$$
r=1.1 \Delta_{S}
$$

Fortunately, the function which relates $r / \Delta_{S}$ to efficiency of the system is not a fast varying function [2], hence non-optimal cases can be used without great losses.

Inspection of Fig. 4 indicates that there is a phase transition at an angle corresponding to SAW velocity on the aluminum surface. However, the slope of the phase transition is positive, resulting in a negative Schoch Displacement! In this case, the evanescent SAW will exist as a backward traveling wave [10], making it inappropriate for focusing in the forward direction.

\section{EXPERIMENT}

We have built a SAW focusing block from lucite. An operating frequency of $1 \mathrm{MHz}$ was chosen. At this frequency and aluminum as the object material, the optimum radius of block from Table 1 is $40 \mathrm{~cm}$. Instead, we have used a block with a radius of $3 \mathrm{~cm}$ giving a focal length of $1.5 \mathrm{~cm}$. This non-optimum but practical size of the block causes a loss of $16 \mathrm{~dB}$ [2]. The inclination angle of the upper surface is to be 31.4 degrees. We have manufactured a number of focusing blocks with slightly varying angles to determine the optimum excitation angle. For proper insonification of the cylindrical surface a block height of $2.8 \mathrm{~cm}$ is chosen. Note that, the shear wave attenuation in lucite is quite high: $2.1 \mathrm{~dB} / \mathrm{cm}$ at $1 \mathrm{MHz}$ as given in the literature [11].

The shear wave transducer was rectangular in shape and $1.1 \mathrm{~cm}$ by $2 \mathrm{~cm}$ in size. This transducer size with the above mentioned cylinder block gives an f-number of 0.75 . Due to low shear impedance of lucite, it is hard to excite shear waves in it by a high impedance PZT transducer. Moreover, high impedance backing used for the transducer reduces coupling into the lucite. We had to add a quarter-wave matching layer between PZT and lucite. The transducer is able to generate 3 microsecond shear wave pulses at $1 \mathrm{MHz}$.

A straight edge discontinuity on the object surface is placed at the expected focus position under the block. A signal is observed with the predicted time delay. The signal showed the characteristics of a focused wave as the position of the edge was varied with respect to block. Fig. 5 shows the the measured signal amplitude as a function of position of

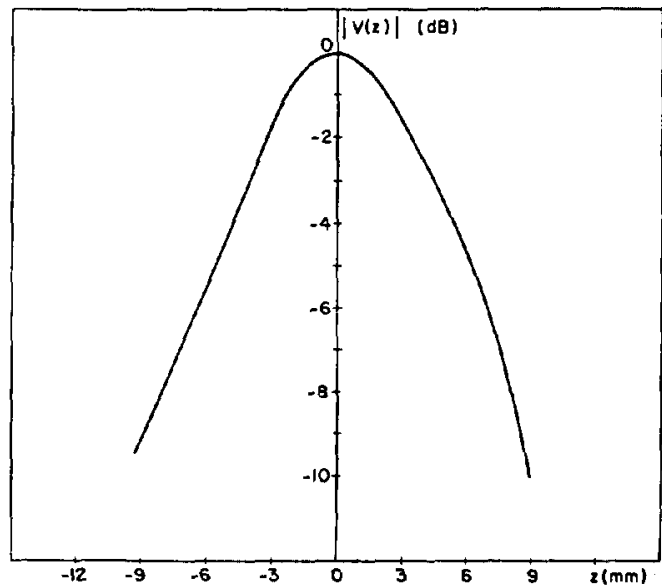

Figure 5: Measured reflected signal amplitude as a function of reflecting edge position with respect to focal point

reflecting edge with respect to the focal point. To be able to calculate the conversion efficiency of the focusing wedge we have measured the signal reflected from a flat lucite block of the same path length with the same transducer. Comparison of this signal level with the signal from the focusing wedge gives a loss value of $16 \mathrm{~dB}$. Comparing this value with the calculated value above shows us that the reflection coefficient of the interface wave at the straight edge discontinuity is close to unity. Note that, we have made no correction for diffraction losses.

To show the focusing ability of the wedge, we placed a series of small holes drilled perpendicular to the surface at the focal line. The modulation depth of the return signal was measured as the focusing block was moved along the focal line. This experiment was repeated for a number of different hole spacings to produce different spatial frequen- 
cies. Fig. 6 is a plot of the results. Experiments indicate that it is able

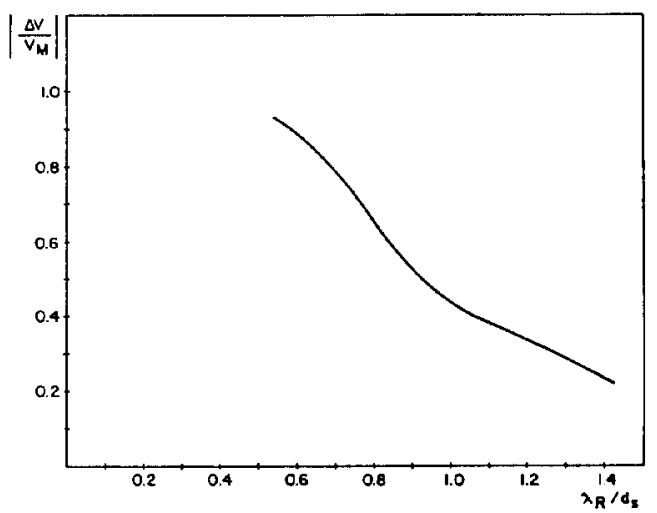

Figure 6: Measured modulation transfer function for the focusing block

to separate holes of $1 \mathrm{~mm}$ diameter with a center-to-center spacing of $2 \mathrm{~mm}$. A resolution of $2 \mathrm{~mm}$ with a surface wave wavelength of $2.5 \mathrm{~mm}$ shows the diffraction limited character of the focused wave.

\section{RESULTS AND CONCLUSIONS}

Non-optimum size of the block causes a rather large loss, $16 \mathrm{~dB}$. This decreases the reflected signal level considerably, but still the signal is easily measurable. An optimal size focusing wedge at low $\mathrm{MHz}$ frequencies is impractically large. At higher frequencies optimality condition can be met, but this time excessive absorption losses will emerge. One may also expect a high level of background signal due to multiple reflections in the bounded geometry of block material. Nevertheless, this background level is significantly lower than the echo signal.

The estimated reflection coefficient of unity for the straight edge discontinuity is not in agreement with a typical SAW reflection coefficient value. For the SAW, a typical reflection coefficient is around 0.4 [12] and it is mainly due to a high transmission coefficient of SAW propagating onto the edge surface. Whereas, for our case a higher reflection coefficient is expected, because the interface wave ceases to propagate when the object surface loses contact with the wedge surface.

At the onset of the project, we expected to be able to excite a SAW on aluminum surface with a velocity very close to the that of the free aluminum surface. However, calculations indicated that only a wave, whose velocity is close to the longitudinal velocity in the lucite, could be excited. This wave has properties very similar to a leaky SAW, but it is a special wave in the sense that it occurs only when two solids are brought together with a slippery bond. This wave velocity changes only very slightly for different object materials as long as the shear wave velocity of the object material is higher than the longitudinal wave velacity of the block material. If the difference of these two velocities are made smaller, the Schoch displacement decreases and consequently, the range of angles in which one can efficiently excite the surface wave will increase. Since the critical angle changes only very slightly, one can build a focusing wedge with a fixed inclination angle applicable to a number of different object materials. If required, a wedge with optimal and practical size may be constructed with proper combination of the block and object materials.

It is difficult to adjust the excitation angle in a solid focusing block, if the Schoch displacement is high. Also, achieving optimal cylinder radius may be difficult. New wedge materials will have to be tried.
For example, calculations indicate that lead would be a proper choice. Not only it is an easy to match material for PZT, but also it results in a small value of Schoch displacement for most materials, hence giving a high efficiency. Lead yields a manageable optimal cylinder radius, which means that $16 \mathrm{~dB}$ loss of lucite wedge can be reduced to the theoretical $3 \mathrm{~dB}$ limit with a wedge made out of lead.

\section{ACKNOWLEDGEMENT}

This work was supported by TUBITAK Ankara Electronics R \& D Institute.

\section{References}

[1] H.Köymen and A. Atalar, "Focusing surface waves with an axicon", Appl. Phys. Lett., vol. 47, pp. 1266-1268, 1985.

[2] A. Atalar and H. Köymen, "Use of a conical axicon as a surface acoustic wave focusing device", IEEE Trans. Ultrason. Ferro. and Freq. Cont., vol. 34 , pp. 53-63, 1987.

[3] H. Köymen, A. Atalar, T. Çiloğlu, M. Önder, Çetin Uzel and H. Yavuz, "Imaging flaws close to surface using focused surface acoustic waves" in Proc. IEEE Ultrasonics Symposium, pp. 755758,1986

[4] H.L. Bertoni and T. Tamir, "Characteristics of wedge transducers for acoustic surface waves", IEEE Trans Sonics Ultrason., vol. 22 , pp. 415-420, 1975.

[5] H.L. Bertoni, "Coupling layers for efficient wedge transducers", IEEE Trans. Sonics Ultrason., vol. 22, pp. 421-430, 1975.

[6] B. A. Auld, Acoustic Waves in Solids, vol. II, Academic Press, New York, 1973.

[7] V.M. Ristic, Principles of Acoustic Devices, John Wiley and Sons, New York, 1983.

[8] L.M. Brekovskikh, Waves in Layered Media, Academic Press, New York, 1960.

[9] H.L. Bertoni and T. Tamir, "Unified theory of Rayleigh angle phenomena for acoustic beams at liquid-solid interfaces", Appl. Phys., vol.2, pp.157-172, 1973

[10] T. Tamir and H.L. Bertoni, "Lateral displacement of optical beams at multilayered and periodic structures", J. Opt. Soc. Am., vol. 61, pp. $1397-1413,1971$.

[11] B. Hartman and J. Jorzysinki, "Ultrasonic hysteresis absorption in polymers", J. Appl. Phys., vol. 43, pp. 4304-4312, 1972.

[12] F.C. Cuozzo, E.L. Cambiaggio, J-P Damiano, and E. Rivier, "Influence of elastic properties on Rayleigh wave scattering by normal discontinuities," IEEE Trans. Sonics Ultrason., vol. 24, pp. 280289,1977 